\title{
Gliadin induced changes in the expression of MHC- class II antigens by human small intestinal epithelium. Organ culture studies with coeliac disease mucosa
}

\author{
S Fais, L Maiuri, F Pallone, M De Vincenzi, G De Ritis, R Troncone, S Auricchio
}

\begin{abstract}
Jejunal biopsies from 16 treated coeliac disease patients and from nine controls were cultured with and without a peptic-tryptic digest of gliadin. Cultures with a peptic-tryptic digest of maize prolamins were also undertaken. Frozen sections of baseline and cultured mucosa were stained by immunofluorescence with an antiHLA-DR monoclonal antibody. Before culture the villous epithelium from both controls and treated coeliac disease expressed DR molecules while the crypt epithelium did not. When biopsies from treated coeliac disease were cultured with gliadin the expression of $D R$ was enhanced in the crypt epithelium in eight of 14 cultures and in 11 of 14 was reduced or absent on the villous epithelium. No change was observed in control cultures. We conclude that gliadin is capable of inducing HLA-DR on the crypt epithelium of in vitro cultured coeliac disease mucosa, providing indirect evidence that gliadin may activate cell mediated immune mechanisms within the small bowel mucosa. This model could prove useful in identifying the immunogenic sequence(s) of gliadins and related prolamines.
\end{abstract}

There is evidence that cell mediated immune mechanisms play a role in the pathogenesis of mucosal damage in coeliac disease and it is suggested that deranged immune response to gliadin occurs in these patients. ${ }^{1}$ This suggestion also seems supported by analogies with animal models. ${ }^{2}$

An altered distribution of the major histocompatibility complex class II molecules on the jejunal epithelium is a relevant feature of both experimental conditions and human coeliac disease. ${ }^{23}$ In the normal human small intestine HLA-DR (DR) is expressed by the villous enterocytes, while epithelial cells in the Lieberkühn crypts do not express this marker. ${ }^{3-5}$ In patients with untreated coeliac disease a markedly enhanced HLA-DR expression occurs in the crypt epithelium, while in treated coeliac disease the pattern of DR epithelial expression is normal. ${ }^{+8}$

These changes are believed to reflect an activated local immune response to gliadin. The aminocacid sequence(s) of gliadin peptides, however, recognised by mucosal antigen specific $T$ cells have not been elucidated. It is yet to be established whether the HLA-DR epithelial distribution observed in coeliacs is caused by the mucosal contact with gliadin. One of the reasons for this is the noticeable lack of in vitro standardised methods in the assessment of mucosal cell mediated immunity to a specific antigen in the human gut.

In the present study we have explored whether gliadin peptides are capable of inducing changes in the epithelial HLA-DR distribution in organ cultures of treated coeliac disease mucosa. We used an organ culture technique as an in vitro system, and the expression of HLA-DR molecules on the crypt epithelium as a marker of activated immunity, to establish an in vitro model for the study of mucosal cell mediated immunity to gliadin in coeliac disease.

\section{Methods}

BIOPSIES

Intestinal biopsies were obtained at the duodenojejunal flexure from 16 coeliac patients on a gluten free diet. All these patients had shown a good clinical and histological response to a gluten free diet for at least 12 months. Nine patients who underwent a jejunal biopsy with no evidence of small intestinal disease were included. Three of them were first degree relatives of coeliac patients; one had a diagnosis of giardiasis and in five the final diagnosis was that of functional bowel disorder.

\section{ORGAN CULTURE TECHNIQUE}

Immediately after excision, each biopsy was placed in $0 \cdot 15 \mathrm{M}$ sodium chloride and examined with a dissecting microscope. Each biopsy was sliced into three approximately equal sized pieces using a scalpel. One piece was fixed in $10 \%$ buffered formalin, embedded in paraffin and cut in $5 \mu$ sections. These sections were stained with haematoxylin and eosin and used for diagnosis. In each specimen intraepithelial lymphocytes were counted and the data expressed as number of intraepithelial lymphocytes per 100 epithelial cells. ${ }^{9}$ The second piece was immediately snap frozen in liquid nitrogen. The remaining piece was placed on a stainless steel mesh, positioned over the central well of an organ culture dish (Falcon, USA) with the villous surface of the biopsies uppermost. The well was then filled with culture medium at $37^{\circ} \mathrm{C}$ so as just to reach the cut surface of the biopsies. Ten millilitres of culture medium consisted of Trowell's T8 medium $(6.5 \mathrm{ml})$, NCTC 135 medium $(2 \mathrm{ml})$, fetal calf serum $(1.5 \mathrm{ml})$, penicillin $50000 \mathrm{IU}$ and streptomycin in $5000 \mathrm{IU}$. The dishes were placed in a sterile 
anaerobic jar which was gassed with $95 \%$ oxygen/ $5 \%$ carbon dioxide for 30 minutes before being sealed and incubated at $37^{\circ} \mathrm{C}$. Biopsies were incubated in medium with or without the addition of a peptic tryptic digest of wheat gliadin at the final concentration of $0.1 \mathrm{mg} / \mathrm{ml}$. The digest was prepared as described elsewhere. ${ }^{10}$ In some experiments the biopsies were also cultured in the presence of peptic tryptic digest of maize prolamins, at the same final concentration. After 30 hours in culture the tissue was snap frozen in liquid nitrogen and stored at $-80^{\circ} \mathrm{C}$.

\section{STAINING TECHNIQUE}

Five micrometre cryostat sections were air dried for four hours, fixed in acetone for $\mathbf{1 0}$ minutes and stored at $-20^{\circ} \mathrm{C}$. Sections were incubated with an undiluted mouse monoclonal antibody to a nonpolymorphic DR determinant (clone L 243, Becton-Dickinson, USA) for two hours at $4^{\circ} \mathrm{C}$. A FITC-conjugated goat antimouse immunoglobulins (Becton-Dickinson, USA) diluted 1:30 was used as a second layer for 30 minutes at room temperature. Sections were washed, mounted in glycerol and the slides were coded in order to make sure that the observer was unaware of the culture conditions. Coded slides were examined with a Leitz Laborlux 12 fluorescence microscope.

\section{Results}

HLA-DR EXPRESSION IN BIOPSY SPECIMENS BEFORE CULTURE

Jejunal biopsies from controls and treated coeliac patients showed a normal mucosal histology. In all cases intraepithelial lymphocytes counts were below 40/100 epithelial cells (range 16-39). When specimens were stained with the antiHLA-DR antibody, the villous epithelium was heavily stained and the maximal fluorescence was detected at the top of the villi. The staining appeared to decrease at the bottom of the villi and was absent in the crypts. The fluorescence was more intense in the subapical and basolateral regions of the enterocytes.

HLA-DR EXPRESSION IN BIOPSIES AFTER CULTURE When biopsy specimens were cultured in medium without gliadin peptides the pattern of epithelial HLA-DR distribution was similar to that observed before the culture (Table) (Fig 1). When the biopsies of treated coeliac patients were cultured in the presence of gliadin peptides, in eight of 14 specimens an increased HLA-DR expression was found in the crypt epithelium

Occurrence of HLA-DR on the villous and crypt epithelium of jejunal biopsies cultured with or without gliadin peptides

\begin{tabular}{llllllll}
\hline & \multicolumn{3}{c}{$\begin{array}{l}\text { Medium + gliadin } \\
\text { digest }\end{array}$} & \multicolumn{2}{l}{$\begin{array}{l}\text { Medium } \\
\text { digest }\end{array}$} \\
\hline & Surface & Crypts & Surface & Crypts & Surface & Crypts \\
\hline Controls & $8 / 8$ & $0 / 8$ & $9 / 9$ & $0 / 9$ & ND & ND \\
Coeliacs & $5 / 6$ & $0 / 6$ & $3^{\star} / 14$ & $8 / 14$ & $7 / 8$ & $0 / 8$ \\
\hline
\end{tabular}

* patchy distribution; $\mathrm{ND}=$ not done.
(Fig 2). In 11 of 14 biopsy specimens, after culture, the expression of HLA-DR in the villous epithelium was markedly reduced or even absent (Fig 3).

Neither the enhanced HLA-DR expression in crypt cells, or its disappearance from villous surface was observed in eight biopsies from treated coeliacs cultured in the presence of maize peptides (Table). No change in the HLA-DR epithelial expression was observed in control specimens cultured in the presence of gliadin peptides (Table).

\section{Discussion}

The organ culture of human small intestine has been proposed as an in vitro model of coeliac disease. In most studies, biopsy specimens from patients on a gluten containing diet have been used. Such specimens showed morphologic and biochemical improvement when cultured in a medium free of gliadin, while no improvement was shown when gliadin was present in the culture medium. " On the contrary, the mucosal morphology of biopsies from coeliac disease on a gluten free diet was not affected by the presence of gliadin in the culture medium. ${ }^{12}$ In the present study we have used cultures of treated coeliac disease mucosa to determine whether changes in the DR epithelial expression could be induced by gliadin even in the absence of obvious mucosal damage.

We did not observe changes in the mucosal histology of specimens from treated coeliacs cultured in the presence of gliadin peptides. Nevertheless, the exposure to gliadin caused changes in the pattern of DR epithelial distribution reproducing the changes found 'in

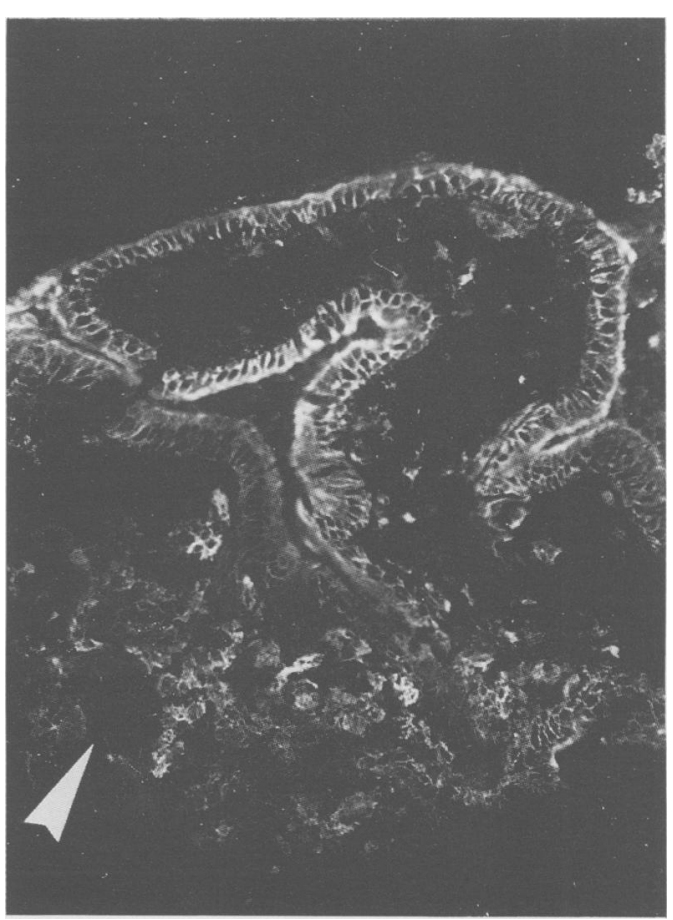

Figure 1: Immunofluorescence staining of HLA-DR determinants in the jejunal mucosa from a patient with treated coeliac disease in vitro cultured in the absence of gliadin peptides. Epithelial cells (enterocytes) on the villi are stained with the anti-HLA-DR antibody while the crypt epithelium (arrows) is negative. Lamina propria contains scattered HI.ADR positive cells. 


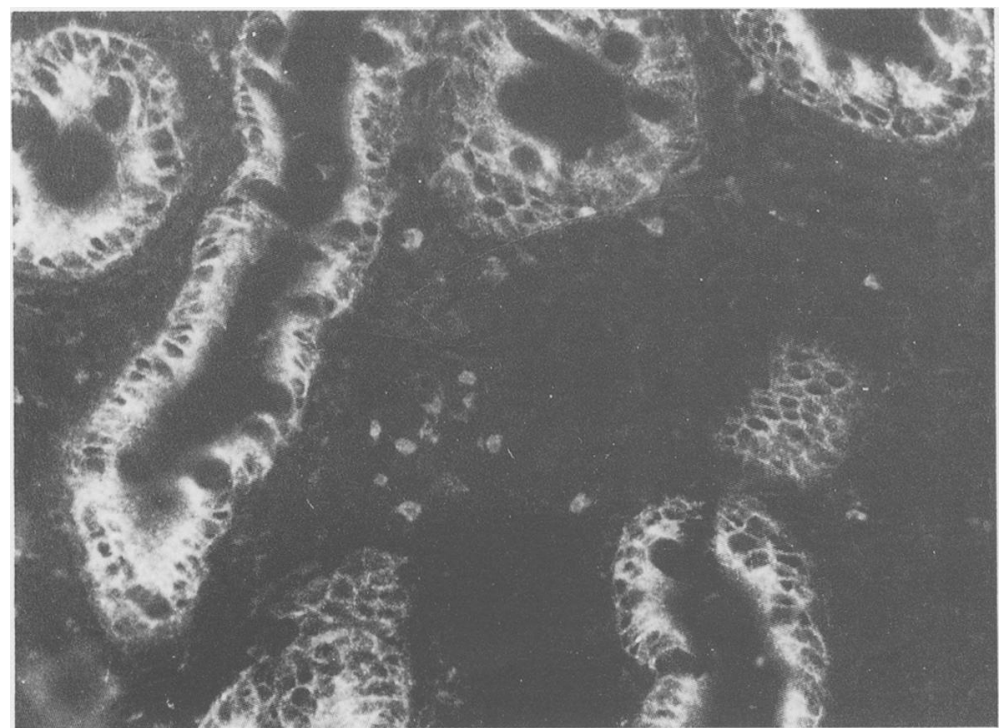

Figure 2:

Immunofluorescence staining of $H L A-D R$ determinants in the jejunal mucosa from a patient with treated coeliac disease in vitro cultured in the presence of gliadin peptides. HLA-DR is uniformly expressed by the crypt epithelium.

Figure 3:

Immunofluorescence staining of $H L A-D R$ determinants in the jejunal mucosa from a patient with treated coeliac disease in vitro cultured in the presence of gliadin peptides. Villous epithelium is negative for $H L A-D R$ with only few weakly positive cells on the top. There are scattered HLA$D R$ positive cells in the villous lamina propria. vivo' in untreated coeliac disease. These changes consisted of an enhanced DR expression by the crypt epithelium and a reduced or absent DR expression by the villous epithelium. The induction of these changes was restricted to mucosal samples of treated coeliacs. The induction of DR in the crypts and loss of epithelial DR from the villi may have different explanations.

As in vivo challenge makes the small intestine sensitive to the in vitro damaging effect of gliadin peptides, "it could be suggested that the biopsies in which DR was induced in the crypts were those from patients in whom the reaction had already been triggered in vivo by small amounts of gluten in the diet. This would not be supported by our finding of a normal mucosal histology and of normal preculture intraepithelial lymphocytes counts in all samples. The relation between the mucosal $\mathrm{T}$ cell activation and the changes in the epithelial DR expression is poorly understood. In animal models class II negative epithelial cells have been induced to express DR when cultured with supernatants of ConA stimulated spleen cells or intraepithelial lymphocytes, ${ }^{13}$ and in man there is indirect evidence that intraepithelial

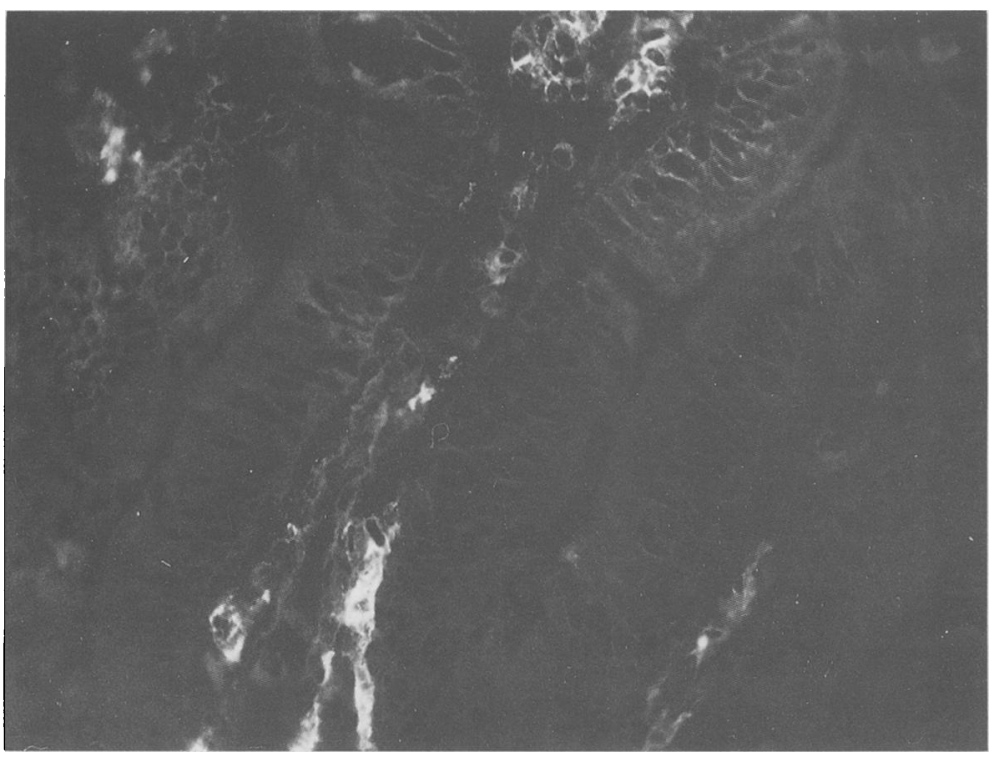

lymphocytes modulate DR expression by enterocytes. ${ }^{x}$ On the other hand there are data available indicating that in coeliac disease the jejunal lamina propria $T$ cells are activated. ${ }^{14}$ In other disease states an enhanced DR expression in the gut epithelium has been found to be significantly associated with an increased number of activated lamina propria lymphocytes capable of releasing the DR inducer interferon gamma. ${ }^{15-18}$ Thus, the activation of gliadin specific $T$ cells within the jejunal lamina propria may have a role in the enhanced crypt cells DR expression in coeliac disease. This seems further supported by the finding of an increased proportion of DR + lamina propria mononuclear cells in the sections of specimens cultured with gliadin (data not shown). Whether these gliadin induced DR + crypt cells may contribute to the antigen-specific mucosal immune reaction in coeliac disease remains to be established. ${ }^{1+19-20}$

The mechanism underlying the disappearance of DR molecules from the villous epithelium during the organ culture is not clear. The biosynthesis of some brush border hydrolases has been shown to be severely decreased by culturing the histologically normal coeliac intestine in the presence of gliadin peptides ${ }^{21}$ and the disaccaridases activity of jejunal mucosa is altered 'in vivo' by the instillation of gliadin suspensions in the duodenum. ${ }^{22}$ It is possible that gliadin interferes with the protein synthesis machinery of the coeliac intestine villous enterocytes, and/or with the transport of glycoproteins to the brush border. The results presented in this paper propose an in vitro model which may prove useful in the identification of the gliadin epitopes involved in the activation of the specific mucosal cell-mediated immune response. Putative toxic sequences of the gliadin have already been identified in studies based on organ cultures of mucosa from patients on a gluten containing diet,,$^{23}$ and the corresponding synthetic peptides are now available. It would be particularly relevant to assess if these peptides are also able to initiate a specific immune response, or if different gliadin peptides are responsible for the activation of mucosal cell mediated immunity, and the in vitro toxicity for the untreated coeliac mucosa.

The authors thank Mr M Termine and Miss Alessandra Piconi (Gastroenterology Unit, Rome), and Mr F Paparo (Pediatric Clinic, Naples, Institute for Alimentary Science, National Clinic, Naples, Institute for Alimentary Science, National Research Council) for technical assistance. The study was
supported by grants from the Italian Ministry of Public Health and
from the Italian National Research Council (Program of Preventive from the Italian National Research Council (Program of Preventive
Medicine, project of Perinatal Pathology and Their Consequences).

1 Auricchio S, Greco L, Troncone R. Gluten sensitive enteropathy in childhood. Pediatr Clin North Am 1988 35: 157-87.

2 Ferguson A. Models of immunologically driven small intestin damage. In: MN Marsh, ed. Immunopathology of the small intestine. Chichester: John W. Immunopathology of the smoth

Bland P. MHC class II expression by the gut epithelium. Immunol Today 1988; 9: 174-8.

4 Arnaud-Battandier, Cerf-Bensussan N, Amsellem R, Schmit: $\mathrm{J}$. Increased HLA-DR expression by enterocytes in children with celiac disease. Gastroenterologv 1986; 91: 1206-12.

5 Ciclitira PJ, Nelufer JM, Ellis HJ, Evans DJ. The effect of gluten on HLA-DR in the small intestinal epithelium of patients with coeliac disease. Clin Exp Immunol 1986; 63: patients

6 Arato A, Savilahti E, Tainio VM, verkasalo M, Klemola T. HLA-DR expression, natural killer cells and IgE containing cells in the jejunal mucosa of coeliac children. (iut 1987; 28 .

7 Marley NJR, Macartney JC, Ciclitira PJ. HIA-IDR, DP and DQ expression in the small intestine of patients with coeliac disease. Clin Exp Immunol 1987; 70: 386-93. 
8 Scott H, Sollid LM, Fausa O, Brandtzaeg P, Thorsby E. Expression of major histocompatibility complex class II subregion products by jejunal epithelium in patients with coeliac disease. Scand f Immunol 1987; 26: 563-71.

9 Ferguson A, Murray D. Quantitation of intraepithelial lymphocytes in human jejunum. Gut 1971; 12: 988-94.

10 Auricchio S, De Ritis G, De Vincenzi M, Occorsio P, Silano V. Effects of gliadin peptides prepared from hexaploid and betraploid wheat on cultures of intestine from rat fetus and coeliac children. Pediatr Res 1982; 16: 1004-10.

11 Jos J, Lenoir G, De Ritis G, Rey J. In vitro pathogenetic studies of coeliac disease. Effects of protein on coeliac intestinal biopsy specimens maintained in culture for 48 intestinal biopsy specimens maintained in

12 Falchuk ZM, Gebhard RL, Sessoms C, et al. An in vitro model of gluten-sensitive enteropathy. Effect of gliadin on intestinal epithelial cells of patients with gluten-sensitive enteropathy in organ culture. $\mathcal{F}$ Clin Invest 1974; 53: 487-92.

13 Cerf Bensussan N, Quaroni A, Kurnick JT, Bhan AK. Intraepithelial lymphocytes modulate Ia expression by intestinal epithelial cells. F Immunol 1984; 132: 2244 52 .

14 Griffiths CEM, Barrison IG, Leonard JN, Caun $\mathrm{K}$, Valdimarsson H, Fry L. Preferential activation of CD4 T lymphocytes in the lamina propria of gluten-sensitive enteropaty. Clin Exp Immunol 1988; 72: 280-3.

15 Fais S, Pallone F, Squarcia O, et al. HLA-DR antigens on colonic epithelial cells in inflammatory bowel disease. Relation to the state of activation of lamina propria lymphocytes and to the epithelial expression of other surface markers. Clin Exp Immunol 1987; 68: 605-12.
16 Pallone F, Fais S, Squarcia O, Biancone L, Pozzilli P, Boirivant M. Activation of peripheral and intestinal lamina propria T lymphocytes in Crohn's disease. In vivo state of activation and in vitro response to stimulation as defined by the expression of early activation antigens. Gut 1987; 28: 745-53.

17 Pallone F, Fais S, Capobianchi MR. HLA-D region antigens on isolated human colonic epithelial cells: enhanced expression in inflammatory bowel disease and in vitro induction by different stimuli. Clin Exp Immunol 1988; 74: $75-9$.

18 Fais $\mathrm{S}$, Capobianchi MR, Pallone F, et al. Spontaneous release of interferon $\gamma$ by intestinal lamina propria lymphocytes in Crohn's disease. Kinetics of in vitro response to interferon $\gamma$ inducers. Gut 1991; 32: 403-7.

19 Bland PW, Warren LG. Antigen presentation by epithelial cells of the rat small intestine. I. Kinetics, antigen specificity and blocking by anti-Ia antisera. Immunology 1986; 58: 1-7.

20 Mayer L, Shlien R. Evidence for function of Ia molecules on gut epithelial cells. F Exp Med 1987; 166: 1471-83.

21 Naim HY, Ambhul P, Sterchi EE, Gaze H, Lentze MJ. As in vitro model system for studying the biosynthesis of brush border membrane (BBM) hydrolases in coeliac disease. Pediatr Res 1987; 22: 102.

22 Mantzaris GJ, Karajannis JA, Jewell DP. The in vivo toxicity of a synthetic dodecapeptide from A-Gliadin. Gastroof a synthetic dodecapept

23 De Ritis, Auricchio S, Jones HW, Lew EJ, Bernardin JE, Kasarda DD. In vitro (organ culture) studies of the toxicity of specific A gliadin peptides in celiac disease. Gastroof specific A gliadin peptid
enterology 1988; $94: 41-9$. 